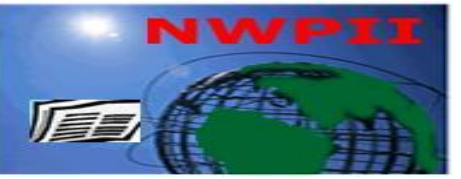

American Journal of Biomedical Sciences

ISSN: 1937-9080

nwpii.com/ajbms

\title{
Targeting Nitric Oxide Mediated Upregulation of Membrane-bound Glucose Regulated-protein 78 by Subtractive Single Chain Variable Fragment (scFv) Phage Display
}

\author{
Lizhi Liu and Weiming $\mathrm{Xu}{ }^{*}$ \\ Department of Molecular Biology and Biotechnology, The University of Sheffield, Firth Court, Western park, \\ Sheffield, UK \\ *Corresponding author: \\ Dr. Weiming Xu \\ Department of Molecular Biology and Biotechnology \\ The University of Sheffield \\ Firth Court, Western park, \\ Sheffield, S10 2TN, UK \\ Telephone: 441142222785 \\ Fax: 441142222800 \\ Email: weiming.xu@sheffield.ac.uk
}

Received: 15 May 2009; | Revised: 28 May 2009; | Accepted: 2 June 2009

\begin{abstract}
Nitric oxide (NO) is a pleiotropic signaling molecule, and may be a contributing factor to the hypoxia encountered in a variety of solid tumors. Previously we reported that increased NOS expression resulted in a concomitant increase in expression of the glucose-regulated protein 78 (GRP78) in human embryonic kidney 293 cells. In the present study, we found that GRP78 was expressed on the cell membrane after nitric oxide induction. In order to isolate membrane-bound GRP78, we have designed a novel scFv library screening method, based on alternative screening with recombinant protein and cell surface-expressed antigen. After first routine screen using GRP78 protein, we used NO-induced cell surface GRP78 as a bait to subtract the none-membrane binding phages. We have successful isolated a panel of scFv specific for GRP78. Treatment of cells with one of the scFv (scFv-GRP78-H19) attenuated the GRP78-mediated protection against thapsigargin, a selective ER $\mathrm{Ca}^{2+}$-ATPase inhibitor. Furthermore, it synergized with ionomycin (IM) to promote apoptosis in prostate cancer cells and inhibited the glucose-deprivationmediated etoposide resistance in human colon and prostate cancer. The work presented here has highlighted the possibility that anti-GRP78 antibodies have the potential to be used therapeutically for the treatment of cancer.
\end{abstract}

Keywords: Nitric oxide; scFv; GRP78; Cancer; Drug resistance. 


\section{Introduction}

In recent years, monoclonal antibodies (MAbs) have become accepted tools for the detection and treatment of cancer [1, 2]. Unmodified antibodies have been approved for the treatment of a variety of cancers including non-Hodgkins lymphoma (Rituximab), breast cancer (Trustuzumab), acute myeloid leukaemia (Gemtuzumab), B-cell chronic lymphocytic leukaemia (Alemtuzumab) and colorectal cancer (Cetuximab, and Bevacizumab). Although the targets for therapeutic antibodies are diverse, they share several important characteristics in that they are expressed on tumor cells but not on healthy tissue, and are surface exposed to be easily targeted by the therapeutic antibody. The mechanism by which antibodies bring about tumor cell death are often complex, but can include recruitment of effector elements (such as complement, or cell-mediated killing processes), or by interacting with receptor-ligand signaling pathways that may result in apoptosis. The traditional method for raising tumor-specific antibodies has been to immunize mice with tumor cells and then to screen for specific MAbs. However, direct selecting the tumor cell surface antigen remains a very difficult task, especially by using tumor cells directly screening naïve phage display libraries due to non-specific cell surface binding of phages, considerable time-consuming proteomic analysis follow-up work and selection of dominant clones $[3,4]$.

We have been examining the biological consequences arising from the expression of nitric oxide synthase (NOS) activity in a cell [5]. NOS activity has been reported in both cancer cell lines and in tumor biopsies, and while high concentrations of NO appear to have anti-tumor properties, lower concentrations are thought to promote tumor growth and neovascularisation [6]. Exposure of a cell to NO has been shown to alter the expression of the cellular transcriptome. In order to study this process systematically, we have developed two regulated cell expression systems where inducible NOS (iNOS) is placed under the control of tetracycline and ecdysoneregulated promoters. Following transfection, the resulting cell lines can generate NO in a dose- and time-dependent manner at concentrations found in biopsies of human tumors [7, 8]. Microarray analysis for both inducible cell lines has shown that NO-mediated regulation can result in the protection of the cells from environmental stress. For example, up-regulation of the DNA-dependent protein-kinase catalytic subunit (DNA-PKcs) is involved in protecting cells from DNA damage[7] while up-regulation of GRP78 [9] is involved in protection against endoplasmic reticulum (ER)stress. These findings have led us to suggest that exposure of cells to sub-lethal 'priming' with NO results in not only protection against higher concentrations of $\mathrm{NO}$ but also protection from other cytotoxic anti-tumor agents. Consequently, NO-regulated genes that are also up-regulated in tumor cells may be good targets for anti-tumor therapy. To test this approach, we decided to target GRP78, as it can be found on the surface of tumor cells [10-12] and provide ER stressmediated cytoprotection in cancer cells [13].

\section{Materials and Methods}

\subsection{Cell culture and immunocytohistochmistry}

EcR293 clone-11 subclone-1 cells have been described previously [9]. Cancer cell lines, PC3(human prostate cancer) and HT-29(human colon cancer) were obtained from the European Collections of Cell Culture. The cells were stained either with an affinity-purified goat polyclonal antibody against the N-terminal peptide of GRP78 (Santa Cruz Biotechnology, CA) or with scFvGRP78-H19. For staining with the anti-peptide antibody, pre-immune goat sera was used as a negative control. For staining with the $\mathrm{scFv}$ antibody, slides staining without first antibody were used as a negative control. A standard Vecta-stain 'ABC kit' (Vector Laboratories, UK) was used with indirect avidin-biotin horseradish peroxidase staining. Indirect immunofluorescence was used to visualize GRP78 protein in the cells. Cells were grown on cover-slips in 6-well plates, washed with PBS, and fixed with PBS/2\% formaldehyde for $30 \mathrm{~min}$ at room temperature. After incubation, the cells were stained with antiGRP78 antibody (Santa Cruz Biotechnology diluted 1:200) or with scFv-GRP78-H19 diluted $(1: 200)$ in PBS. In the case of scFv-GRP78-H19, 
the second antibody was targeted against the cmyc-tag (mouse MAb 9E10, Cambridge Research Biochemicals, Wilmington, DE) in 1:1000 dilutions. Cells were incubated for $45 \mathrm{~min}$ at $37^{\circ} \mathrm{C}$ with FITC-conjugated affinity-purified goat anti-goat IgG or with anti-mouse IgG-Texas-red conjugate diluted 1:100. In some cases, a second antibody against the c-myc-tag fluro555 conjugate (Upstate Ltd, Dundee, UK) was also used (diluted 1:200). After washing three times in PBS, images were captured on a confocal microscope (Leica TCS SP, Germany).

\subsection{Selection of cell surface targeting anti- GRP78-specific scFv by cell surface subtractive screen}

GRP78 protein was purified as described previously [14] and was used to coat immunotubes for $\mathrm{scFv}$ panning experiments and later subtractive screening. The Tomlinson naïve human scFv libraries I + J (15, comprising $10^{8}$ different $\mathrm{scFv}$ fragments, MRC Geneservice, UK) were screened using the protocol recommended by the MRC Geneservice, UK. In the first round immuno-tubes coated with $10 \mu \mathrm{g} \quad \mathrm{ml}^{-1}$ recombinant murine GRP78. Phage repertoires recovered from this step were used in a second round of panning using the whole cell surface as bait. EcR293 clone-11 subclone-1 cells were grown as an attached monolayer in T25 flasks until almost confluent and induced to generate NO by treatment with $10 \mu \mathrm{M}$ muristerone A for $16 \mathrm{~h}$, resulting in a 3-4 fold increase in GRP78 protein [9]. $2 \times 10^{6}$ cells were washed with PBS, and fixed with $4 \%$ paraformaldehyde (Sigma, UK) for $10 \mathrm{~min}$ at room temperature. The fixed cells were washed with PBS and blocked with 2\% MPBS (PBS supplemented with milk powder to a final concentration of $2 \%$ ) for $2 \mathrm{~h}$ at room temperature. Meanwhile, the phage repertoires recovered from the first-round panning against GRP78 protein were separately incubated with 50:50 volume of 4\% MPBS and DMEM medium(Invitrogen, UK). After 2\% MPBS blocking, the cells were incubated with preblocked phage repertoires in 50:50 volumes of 4\%MPBS and DMEM medium for $2 \mathrm{~h}$ at room temperature to isolate anti-GRP78 antibodies that recognised surface-exposed of panning, $1 \times 10^{12}$ phage were added to

epitopes. After incubation, the cells washed rapidly 10 times with $\mathrm{PBS}$ at room temperature. Phage adhering strongly to the cell surface were eluted following treatment with trypsin $\left(1 \mathrm{mg} \mathrm{ml}^{-1}\right.$ in $1 \mathrm{mM} \mathrm{CaCl}_{2}, 50 \mathrm{mM}$ Tris-HCL, $\mathrm{pH} 7.4$ ), and were used to re-infect $E$. coli with help phage TG1. Two more rounds of panning were carried out against recombinant GRP78 $\left(10 \mu \mathrm{g} \mathrm{ml} \mathrm{m}^{-1}\right)$ to isolate specific anti-GRP78 scFv. After the fourth round of panning, a panel of 200 phage was picked and the encoded scFv antibody screened using an enzyme-linked immunosorbent assay (ELISA) against recombinant GRP78 protein (as described in the MRC protocol). ELISA-positive phage were used to re-infect E. coli HB2151 (a non-suppressor strain), and the resulting soluble anti-GRP78 scFv purified using Ni-TED spin column chromatography according the manufacturer's recommended conditions (NiTED, Active Motif Europe, Rixensart, Belgium).

\subsection{Surface Plasmon Resonance}

Surface Plasmon Resonance studies on the binding of scFv-GRP78-H19 to GRP78 were carried out on a Biacore 3000, essentially following the manufacturer's recommended conditions (Biacore, Uppsala, Sweden). Briefly, the GRP78 protein and control protein BSA were immobilized on CM5 chips(Biacore) with surface densities of 200 resonance units(RU) using amine-coupling kit(BIAcore). Antibody injections were performed in a concentration range of 9.37$74 \mathrm{nM}$. Assoication and dissociation data from all concentrations were fit globally using Biaeval 4.1 with 1:1 Langmuir binding model.

\subsection{Cell Surface analysis of GRP78 by flow cytometry}

Cells were fixed with $2 \%$ paraformaldehyde for 30 mins and preincubated with $1 \%$ horse serum for further $1 \mathrm{~h}$. The cells were stained with anti-GRP78 antibody, scFv-GRP78-H19 (1:00 dilution) overnight with slow rotation. After washed with PBS, c-myc-tag (mouse MAb 9E10) in 1:500 dilutions were added the cells for $1 \mathrm{~h}$. After washed with PBS, cells were staining with goat $\mathrm{F}(\mathrm{ab})_{2}$ fragment anti-mouse IgG-FITC (Beckman Coulter, UK). In negative controls, the first antibody scFv-GRP78-H19 was omitted in

Am. J. Biomed. Sci. 2009, 1(4), 321-335; doi: 10.5099/aj090400321 
the staining procedure. At lease $5 \times 10^{3}$ cells were analyzed on a FACSCalibur (BD Biosciences. UK) using Cellquest and FlowJo software.

\subsection{Cell growth assay and cytoprotection assay}

Thapsigargin (TG) and cell viability experiments were carried out essentially as described previously [9]. Briefly, EcR293 clone11 subclone-1 cells were treated with muristerone A $(10 \mu \mathrm{M})$ for $16 \mathrm{~h}$ and were subsequently exposed to TG (37 $\mathrm{nM}$ ) with or without antibody. Cell viability was determined after $48 \mathrm{~h}$ using an LDH cytotoxicity detection kit (Roche, Lewes, UK). To detect apoptosis, PC-3 cells or HT-29 cells were exposed to IM (100 nM) or etoposide $(10 \mu \mathrm{g} / \mathrm{ml})$ in the presence of scFv-GRP78-H19 (83 nM) for 48-76 h. Control treatments were carried out either using unrelated antibody scFvA20 or heat-inactive antibody $\left(100^{\circ} \mathrm{C}, 20 \mathrm{mins}\right)$. For etoposide treatment, 2-Deoxyglucose (2DG, $20 \mathrm{mM}$ ) was added into culture medium for $16 \mathrm{~h}$ before adding etoposide. Detection of nuclear chromatin condensation was carried out with the Hoechst dye H33342 (10 $\left.\mathrm{g} \mathrm{m} \mathrm{m}^{-1}\right)$ for $15 \mathrm{~min}$, and nuclei were visualized using a Leica DMIRB inverted fluorescence microscope. A minimum of 200 cells were counted in each sample and condensed or fragmented nuclei were expressed as a percentage of the total number of nuclei.

\subsection{Detection of Caspase-3/7 activity}

Dual caspase-3/7 activity was determined with an Apo-ONE caspase-3-7 assay kit (Promega, $\mathrm{UK})$ using the manufacturer's conditions. PC-3 cells $\left(1 \times 10^{5}\right.$ cells $\left.\mathrm{ml}^{-1}\right)$ were grown in 6-well plates and exposed to IM (100 nM) alone or in the presence of scFv-GRP78-H19 $(83 \mathrm{nM})$ or the control antibody scFv-A20 for $76 \mathrm{~h}$. Caspase activity was measured as a relative fluorescence unit (RFU) using a SpectraMax Plus spectrophotometer $(485 \mathrm{~nm}$ excitation, $527 \mathrm{~nm}$ emission). Western blot analysis was carried out essentially as previously described [9]. GRP78 antibody was from BD Bioscience, Oxford, UK.

\subsection{Sequencing and Antibody epitope mapping}

Qiagen DNA purification kit was used to isolate phagemids DNAs. The sequence primers for $\mathrm{V}_{\mathrm{H}}$ was 5'- CGACCCGCCACCGCCGCTG and for $\mathrm{V}_{\mathrm{k}}$ was 5'- CTATGCGGCCCCATTCA. For the antibody epitope mapping, human GRP78 $\mathrm{N}$-terminal 20 amino acid synthetic peptides (SC1050P, Santz Cruz, CA, USA) and C-terminal 20 amino acid synthetic peptides (SC-1051P, Santz Cruz, CA, USA) were spotted on nitrocellulose membrane and probed with scFv antibodies. The second antibody was targeted against the c-myctag mouse MAb 9E10, in 1:1000 dilution and a standard Vecta-stain 'ABC kit' was used with indirect avidin-biotin horseradish peroxidase staining. The brown positive staining were scanned and analyzed with Scan analysis (Cambridge, UK) program.

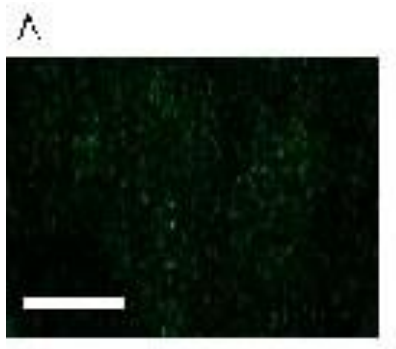

c

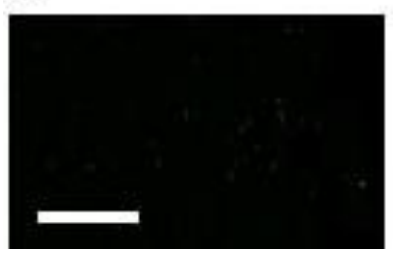

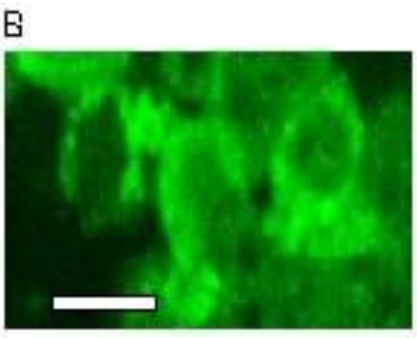

D

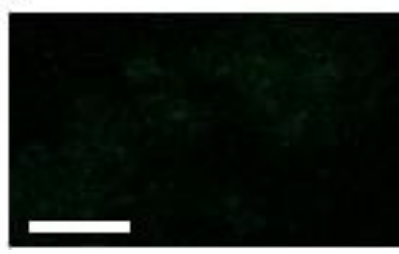

Fig.1 GRP78 protein was identified on the surface of NO-generating EcR293 clone-11 subclone-1 cells after $10 \mu \mathrm{M}$ muristerone A induction for $16 \mathrm{~h}$ [9]. A. Un-induced cells. B. Induced cells using a polyclonal anti-GRP78 antibody (Santa Cruz Biotechnology). C. Control staining using preimmune serum only, un-induced cells. D. Control staining using pre-immune serum only, induced cells. Scale bar represents $20 \mu \mathrm{m}$.

\section{Results}

\subsection{GRP78 is expressed on the cell membrane after nitric oxide induction}

We have shown previously that NO can mediate an increase in GRP78 protein in an ecdysone-inducible human embryonic kidney (EcR293) cell line [9]. Treatment of EcR293 


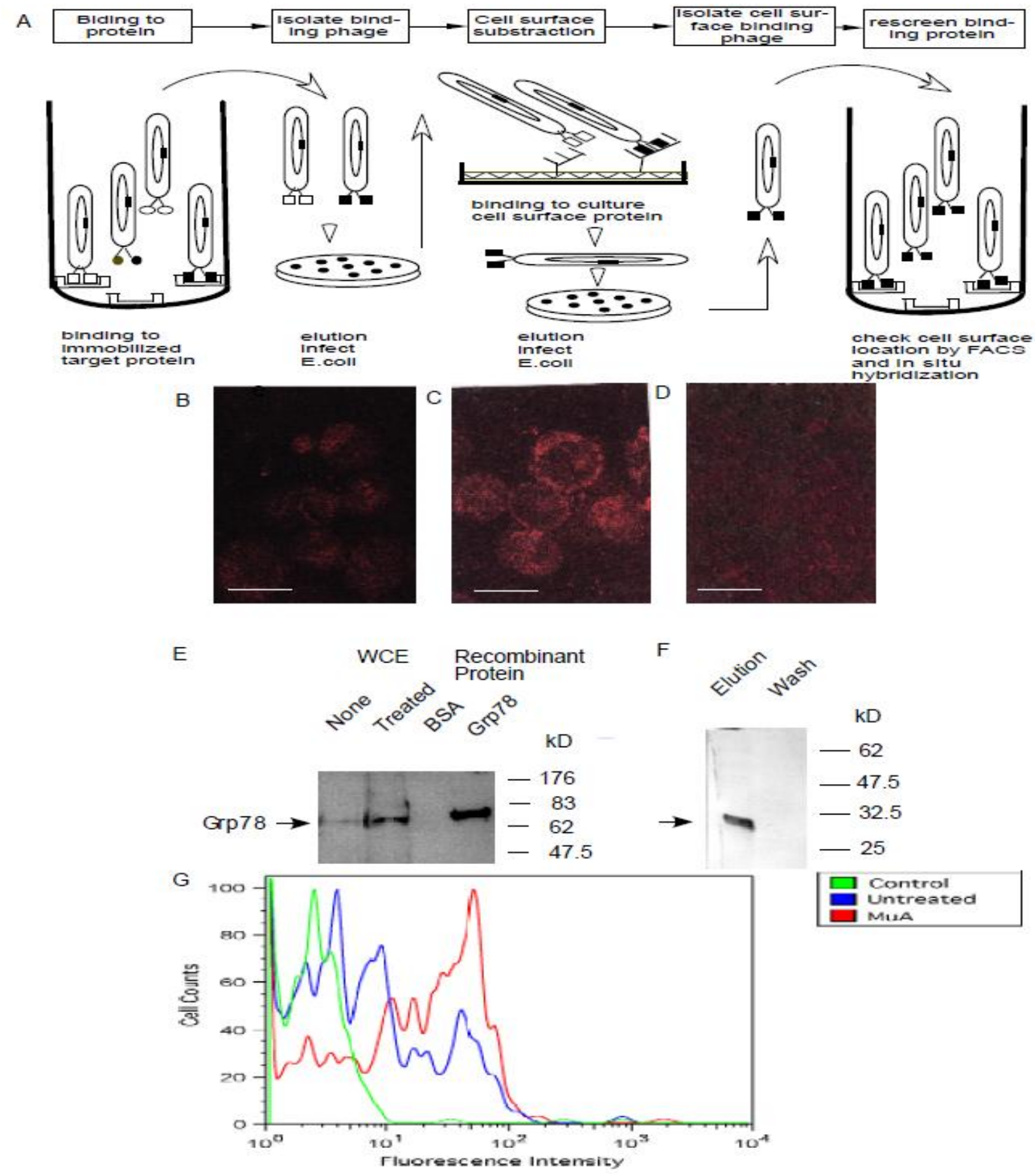

Fig. 2. A. Subtractive-screening using a phage display library. A large collection of human naïve phage is screened first against immobilized recombinant GRP78 protein. The isolate phages are then screened against tissue culture cells which have membranelocated GRP78 protein exposed on the cell surface. These isolated cell surfaces binding phage can then be re-screened with recombinant target protein. B. Immunocytohistochemistry on GRP78 using scFv-GRP78-H19 scFv, with a second antibody against the c-myc-tag and staining with anti-mouse IgG-Texas Red conjugate in EcR293 clone-11 subclone-1 cells. Scale bar represents $20 \mu \mathrm{m}$ in B-D. C. Detecting surface GRP78 protein in NO-generating cells after $10 \mu \mathrm{M}$ muristerone A induction for 16 h [9] with scFv-GRP78-H19. D. Control staining omitting the first antibody; scale bar represents $20 \mu \mathrm{m}$ in B-D. E. Western blot analysis of GRP78 protein using scFv-GRP78-H19. Whole cell extracts (WCE) of EcR293-clone-11 subclone-1 cells with (Treated, track 2) or without muristerone A induction (none, track 1) for $16 \mathrm{~h}$. BSA (track-3) and purified GRP78 (track-4) were used as controls. The western blot represents three independent experiments. F. SDS-polyacrylamide gel electrophoresis analysis of soluble scFv-GRP78-H19. G. Flow cytometric analysis using the scFv-GRP78-H19 antibody. MuA, muristerone A.

Am. J. Biomed. Sci. 2009, 1(4), 321-335; doi: 10.5099/aj090400321 @ 2009 by NWPII. All rights reserved. 
A. Deduced amino acid sequences of the heavy-chiain and light-chiain CDR H3 and CDRL1, CDR-L2 and CDR-L3 regions of the ScFv-Grp78-H19, L21 and L2 clones

\begin{tabular}{|l|llllll|}
\hline Clone & $V_{\mathrm{H} \text { family }}$ & CDRH3 & $\mathrm{V}_{\text {L family }}$ & CDR L1 & CDRL2 & CDRL3 \\
\hline H19 & $V_{\mathrm{H} 3}$ & KINSTKEV & $\mathrm{V}_{\mathrm{L} 1}$ & SGSSSNI & RNNQRPSGV & CAAWDD \\
L21 & $\mathrm{V}_{\mathrm{H} 3}$ & KINNTKEV & $\mathrm{V}_{\mathrm{L} 1}$ & SGSSSNL & RNNHRPSGV & CATWDD \\
L2 & $\mathrm{V}_{\mathrm{H} 3}$ & KINSTNEV & $\mathrm{V}_{\mathrm{L} 1}$ & SGSSSNL & RNNHRPSGV & CAAWDD \\
\hline
\end{tabular}

B

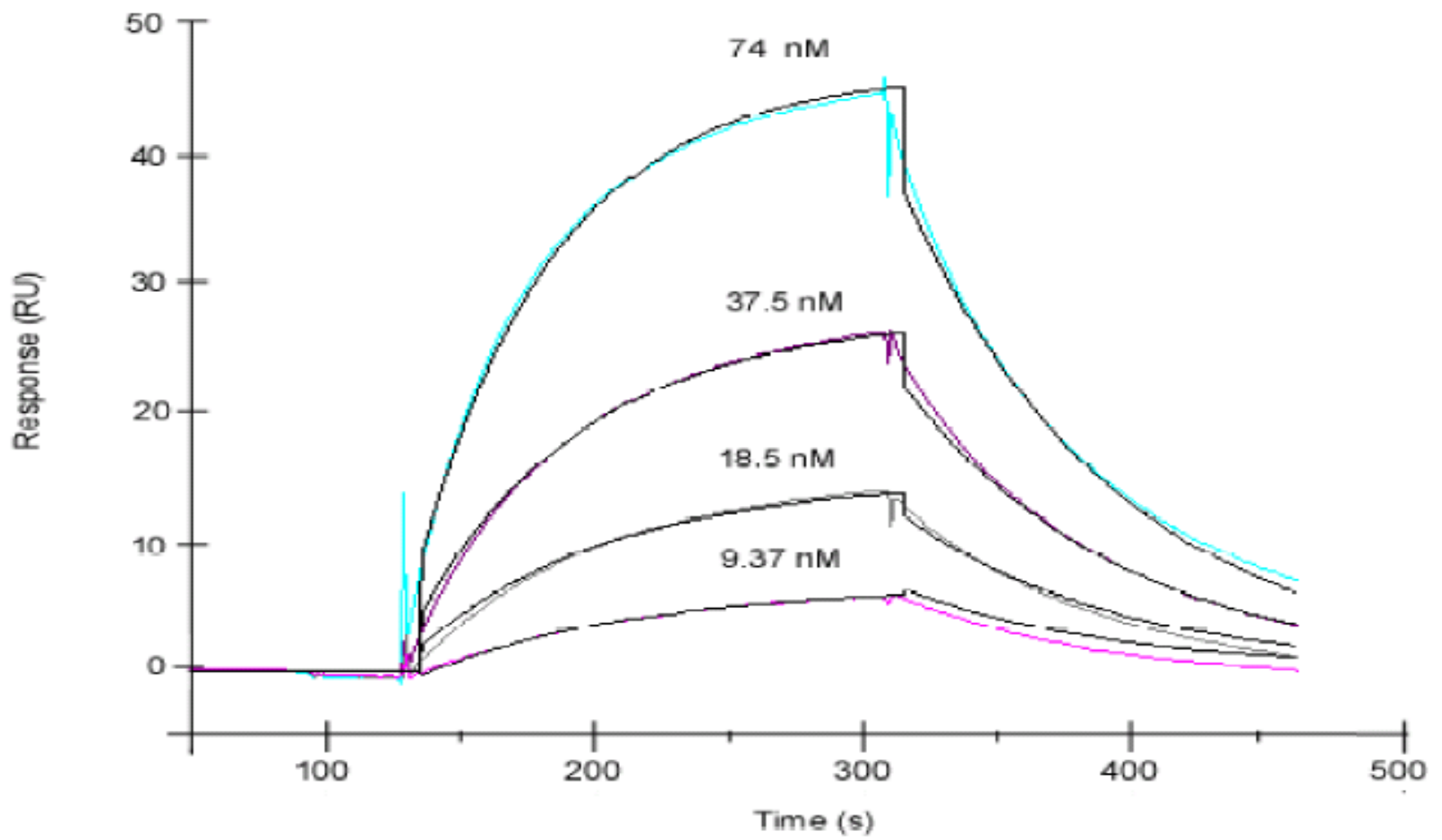

Fig.3 Sequence analysis of the selected GRP78-specific scFv and SPR analysis of scFV-GRP78-H19. A. Deduced amino acid sequence alignments of the $\mathrm{V}_{\mathrm{H}}$ and $\mathrm{V}_{\mathrm{L}}$ regions. B. SPR analysis of interactions of GRP78 and scFV-GRP78-H19 antibody. Representative overlays for various concentrations of scFV antibody to immobilized GRP78 using BSA protein as baseline control. Color lines are data; black lines were indicated a good fit to simple 1:1 kinetic model with a Kd of $17.5 \mathrm{nM}$.

clone-11 subclone-1 cells with $10 \mu \mathrm{M}$ muristerone A for $16 \mathrm{~h}$ resulted in a 3 - to 4 -fold increase in GRP78[9] . Fluorescence immunohistochemistry was carried out on these cells using an antiGRP78 N-terminal antibody and FITCconjugated affinity-purified goat secondary antibody. A strong surface staining of GRP78 protein was observed in NO-generating cells (Fig. 1B) in comparison with un-induced cells (Fig.1A). There was no specific staining in the control cells in which the pre-immune serum was used (Fig. 1C, un-induced and Fig1D, induced). 


\subsection{Isolation of anti-membrane-bound GRP78 scFVs using subtractive-screening in a phage display library}

To isolate antibodies that recognized surfaceexposed GRP78, we decided to use this cell line to subtract the non-cell surface binding GRP78 from human native phage display libraries (Fig.2A). Two human scFv-phage display libraries [15], (Tomlinson I and $\mathrm{J}$ human single fold $\mathrm{scFv}$ libraries) were screened initially against recombinant GRP78 protein [14]. The resulting GRP78-specific phage were pooled and applied to a monolayer of EcR293 clone-11 subclone-1 cells that had been treated with $10 \mu \mathrm{M}$ muristerone $\mathrm{A}$ to express GRP78 on the cell surface [9]. After this whole cell selection step (Fig.2A), the cells washed rapidly 10 times with $\mathrm{PBS}$ at room temperature. Phage adhering strongly to the cell surface was eluted following treatment with trypsin. Around $3 \times 10^{4}$ phage were recovered from $1 \times 10^{11}$ phage repertoires. After this subtractive screen to separate the phages binding to the cell surface to non-cell surface phage binder, two further rounds of screening were carried out against recombinant GRP78 protein. A panel of 200 phage were picked and the encoded scFv antibody screened using an enzyme-linked immunosorbent assay (ELISA) against recombinant GRP78 protein. 19 positive phage have been identified by Elisa assay. Total three different unique clones have been identified by sequencing. One of the antibodies isolated by this procedure, scFv-GRP78-H19, was selected for further study and was shown by immunohistochemistry to detect surface-located GRP78. The antibody showed a strong signal for cell surface GRP78 in the NO-generating cells (Fig. 2C) and a relatively low cytoplasmic staining in un-induced cells (Fig.2B). No specific staining was detected in control samples in which the primary antibody was omitted (Fig. 2D). ScFv-GRP78-H19 was also able to detect GRP78 in western blots of whole cell extracts while it did not cross-react with the negative control bovine serum albumin, BSA (Fig. 2E). Purified soluble scFv-GRP78-H19 has a molecular weight of around $30 \mathrm{kDa}$ (Fig. 2F). Flow cytometric analysis using the antibody revealed a strong cell surface-located GRP78 fluorescence signal in
NO-generating cell (Fig. 2G, Muristerone A treatment), in comparison with uninduced cells. There was little fluorescence present in negative control.

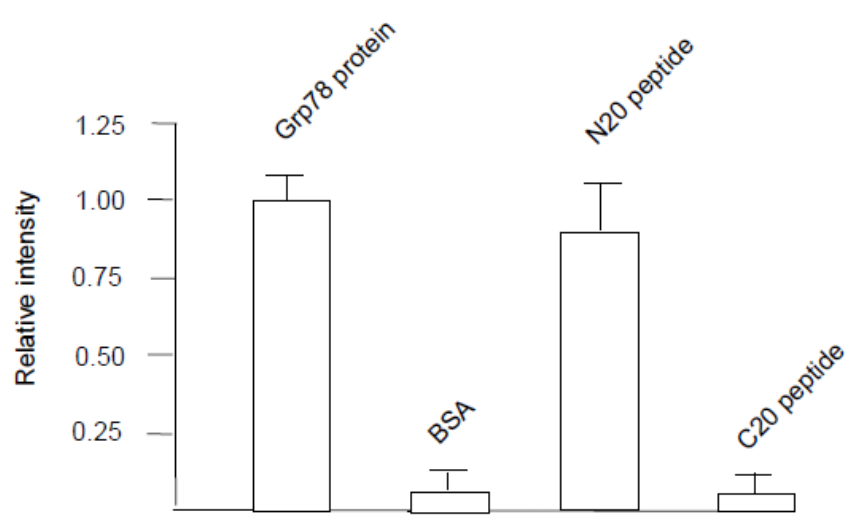

Fig.4. ScFv-GRP78-H19 antibody epitope mapping. Four different proteins or peptides (200 $\mu \mathrm{g} / \mathrm{ml}$ ) were spotted on a nitrocellulose filter and probed with ScFV-GRP78-H19 antibody. The signal intensity of GRP78 protein spot was used as internal control as 1. Only GRP78 N20 peptide (SC-1050P, which cover N-terminal 10-60 amino acids epitope of GRP78) has strong immunodetection signal $(80 \%)$ in comparison with GRP78 protein $(n=3)$.

The deduced amino acid sequences of the variable heavy-chain and the light-chain regions from the three unique selected GRP78-specific scFvs are shown on Fig3A. They all comprise a $\mathrm{V}_{\mathrm{H} 3}$ heavy chain and a $\mathrm{V}_{\mathrm{L} 1}$ light chain (Fig. 3A; data generated using the DNAPLOT and VBASE programs from the MRC Protein Engineering Centre; www.mrc-cpe.cam.ac.uk). Furthermore, scFv-GRP78-H19 was shown to bind to GRP78 in surface plasmon resonance (SPR) experiments with the kinetic constants for dissociation $(\mathrm{Kd})$ at 17.8nM (n=3, BIAevaluation 4.1, Fig.3B). To map the antibody epitope, we have taken advantaged of the immunofluorescence staining pattern of the scFv-GRP78-H19 being very similar as that of anti-human GRP78 N-terminal 20 amino acid synthetic peptide antibody (SC1050, Santz Cruz, CA, USA $)(\mathrm{Xu}$ et al., unpublished data). The immuno-reaction of the antibody to N20 terminal peptides(SC1050P,range from 10-60 amino acids of human GRP78) have similar strong as GRP78 protein, 


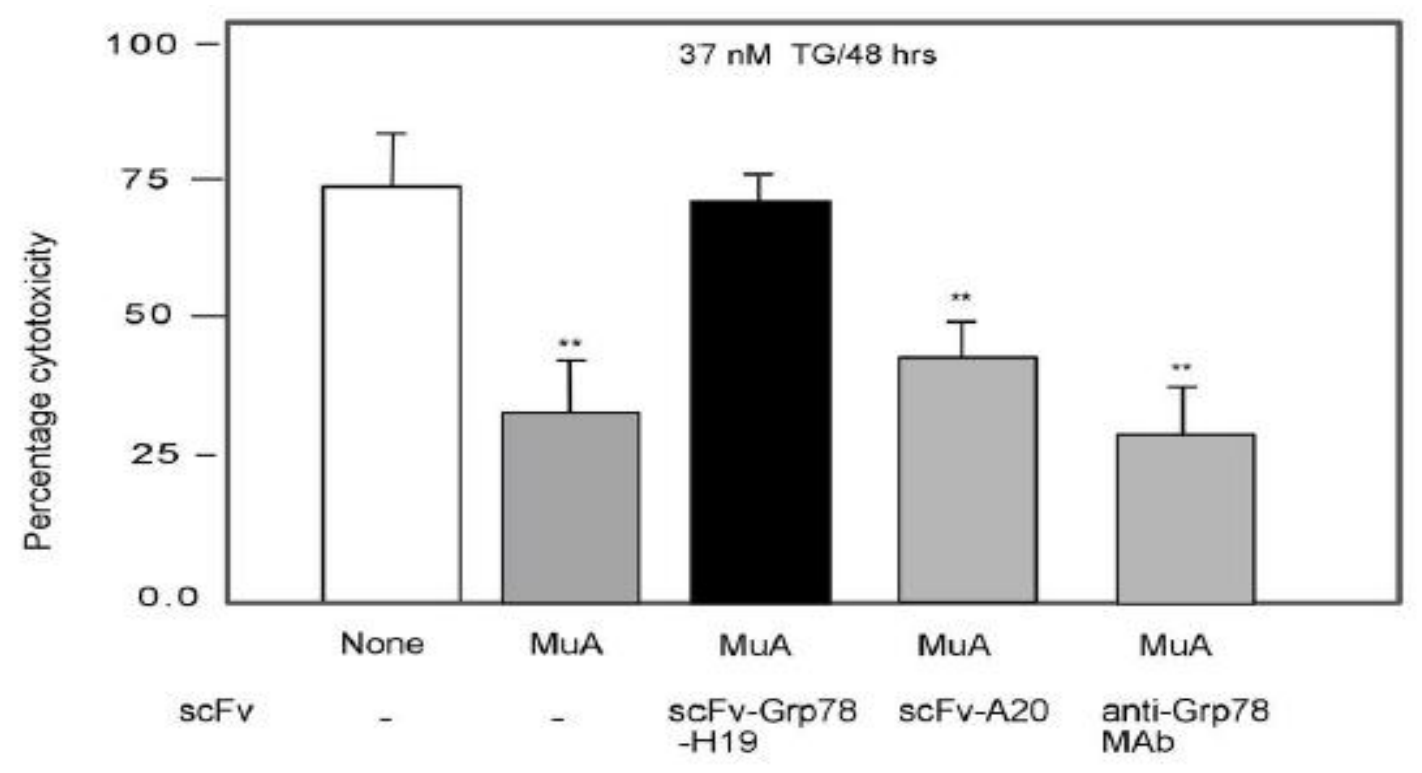

B
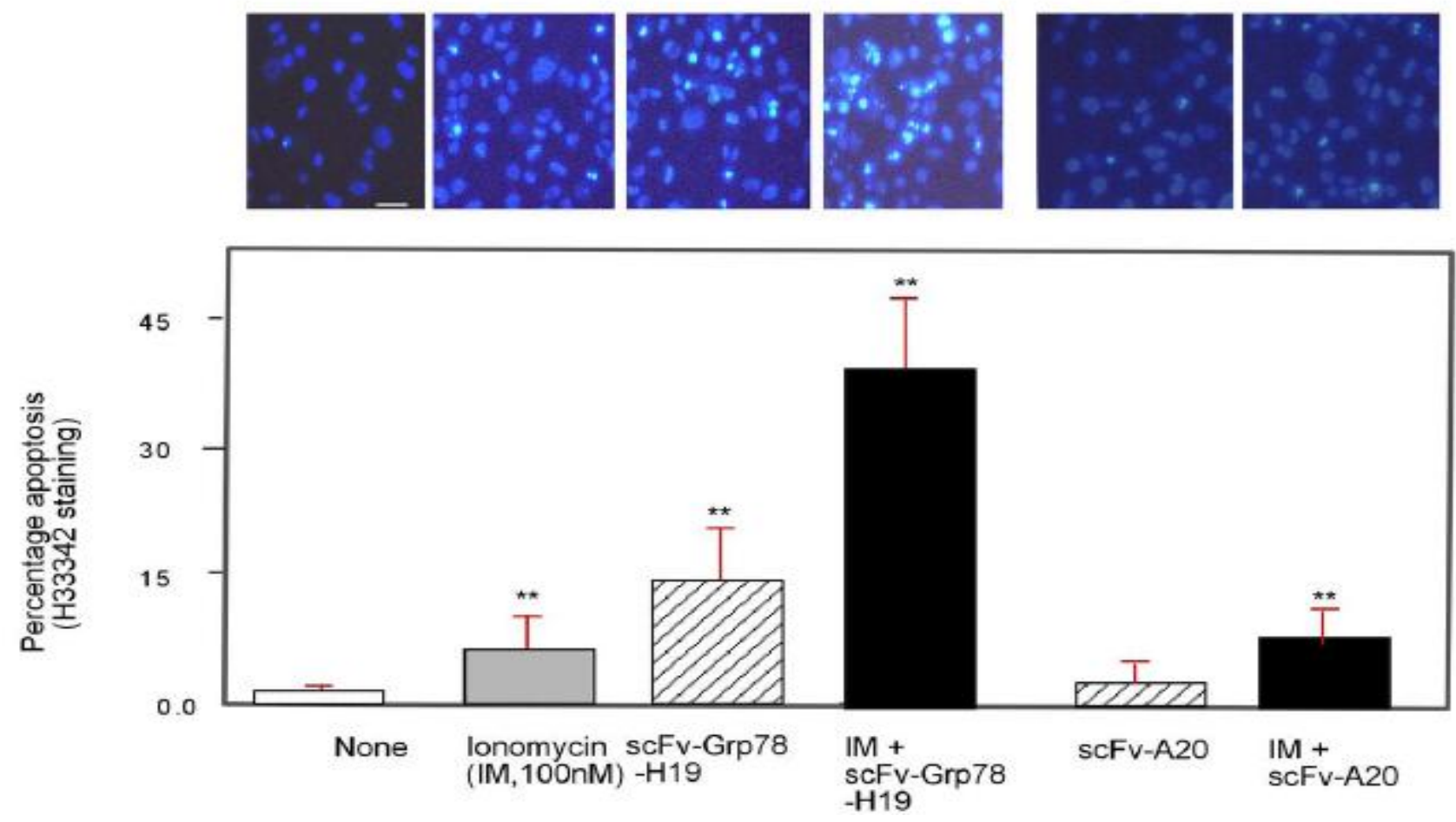

Fig. 5. Effect of scFv-GRP78-H19 on cytoprotection and cell viability. A. EcR293 clone-11 subclone-1 cells incubated in the presence (or absence; None) of muristerone A for $16 \mathrm{~h}$ were washed with PBS, treated with TG (37 $\mathrm{nM}$ ) and with scFv-GRP78-H19, control antibody scFv-A20 or anti-GRP78 MAb (BD Bioscience). Cell viability was determined by the LDH assay after a further $48 \mathrm{~h}$. Values are the mean values \pm S.D. of three replicates. Double asterisk indicates a significant difference $(\mathrm{P}<0.01)$ between NO-generating and non NO-generating cells. B. Effects of scFv-GRP78-H19 on the survival of PC-3 cells treated with ionomycin (IM; $100 \mathrm{nM}$ ) for $72 \mathrm{~h}$. Apoptotic nuclei or nuclear DNA strand breaks were visualized using Hoechst DNA dye H33342. Scale bar represents $20 \mu \mathrm{m}$ on all samples. Values in the graph are the mean \pm S.D. of 3 individual experiments. Double asterisk indicates a significant difference $(\mathrm{P}<0.01)$ between none treatment cells and IM or antibody treatment cells. 
while no immuno-reaction with $\mathrm{C} 00 \mathrm{H}$-terminal GRP78 peptide(SC-1051P) and BSA(Fig4), indicating the antibody epitope located on the $\mathrm{NH}$ (2)-terminal domain of GRP78 at 10-60 amino acids(Fig.4).

\subsection{ScFv-GRP78-H19 and GRP78-mediated cytoprotection}

We have shown previously that NO generation in a cell increased the concentration of GRP78 and resulted in cytoprotection against thapsigargin (TG), a selective ER $\mathrm{Ca}^{2+}$-ATPase inhibitor [9]. To investigate whether treatment with an anti-GRP78 antibody could reverse this protection, EcR293 clone-11 subclone-1 cells were treated with $10 \mu \mathrm{M}$ muristerone to induce NO production and increase the concentration of GRP78. After $16 \mathrm{~h}$, cells were exposed to TG (37 $\mathrm{nM})$ in the presence of either scFv-GRP78-H19 $(83 \mathrm{nM})$ or with the same concentration of a nonGRP78 cross-reactive control antibody (scFvA20, EMBL Access number AM939369). Treatment with scFv-GRP78-H19 (Fig. 5A) abolished completely the NO-mediated cytoprotection at $48 \mathrm{~h}$. The negative control antibody scFv-A20 had no apparent effect on cell survival (Fig. 5A) nor did treatment with $83 \mathrm{nM}$ of a commercially available anti-GRP78 MAb (BD Transduction Labs, Cat 610979).

GRP78 has been shown previously to provide cytoprotection against apoptosis in the prostate cancer cell line PC-3 mediated by the calcium ionophore ionomycin (IM) [16]. To extend our protection studies, PC-3 cells were treated with IM and scFv-GRP78-H19 (83 nM) (Fig.5B). Although treatment with scFv-GRP78-H19 or IM alone produced a low but significant increase in the number of fragmented nuclei and nuclei with DNA strand breaks (judged by Hoechst 33342 staining), a combination of IM and antibody resulted in a significant increase in apoptotic nuclei. The combination of scFv-GRP78-H19 and IM resulted in significantly more apoptotic cell death $(38 \%)$ than treatment with scFv-GRP78H19 (15\%) or IM (7\%) alone after $72 \mathrm{~h}$ (Fig. 5B). Non-GRP78-reactive scFv-20 was used as a negative control.

Immunofluorescence with scFv-GRP78-H19 detected a weak signal of surface-located GRP78 in PC-3 cells (Fig. 6A). Following treatment with IM (100 nM) for $76 \mathrm{~h}$, both surface-located and intracellular GRP78 were increased significantly (Fig. 6B). No specific cell surface or intracellular staining was observed using control antibody scFv-20 following exposure of cells to the same concentration of IM (Fig. 6C).

To investigate the mechanism of scFvGRP78-H19-mediated apoptosis, caspase 3/7 activity was measured following the treatment of PC-3 cells with IM, scFv-GRP78-H19 or a combination of both (Fig. 6D). Treatment of PC3 cells with IM (100 nM) or scFv-GRP78-H19 $(83 \mathrm{nM})$ for $72 \mathrm{~h}$ produced a 1.4-fold and 2-fold increase in caspase-3/7 activity respectively, while the combination of both resulted in a 3-fold increase.

\subsection{ScFv-GRP78-H19 inhibited etoposide drug resistance in colon and prostate cancer cells under glucose deprived condition}

Glucose deprivation-mediated GRP78 increase has been shown previously to provide cytoprotection against etoposide-mediated apoptosis in colon cancer cell line HT-29 under either 2-Deoxyglucose (2DG) treatment or glucose-free medium treatment [17]. To determine whether scFv-GRP78-H19 antibody can attenuate GRP78-mediated drug resistance against etoposide, HT-29 colon cancer cells were treated with etoposide $(10 \mu \mathrm{g} / \mathrm{ml})$ only, etoposide and 2DG(20mM), scFv-GRP78-H1 antibody(83nM) only, scFv-GRP78-H19 antibody plus etoposide and 2DG, heat-inactive scFvGRP78-H1 antibody plus etoposide and 2DG(Fig.7A). We found that after $48 \mathrm{~h}$ treatment, there were $7 \%+2$ cells having apoptotic nuclei and nuclear DNA strand break, visualized using Hoechst DNA dye H33342 in etoposide treated samples (Fig.7A). Such apoptotic cells disappeared after treating cells with 2DG and etoposide, which led GRP78 protein increase (Fig.8). Although treating HT-29 cells with the scFv-GRP78-H19 antibody induced $8 \%+3$ apoptotic cells, there were significant apoptotic cell death $(22 \%)$ in the scFv-GRP78-H19 antibody plus etoposide and 2DG treatment group, indicating the antibody can attenuate etoposide drug resistance in colon cancer cells under

(C) 2009 by NWPII. All rights reserved. 
A

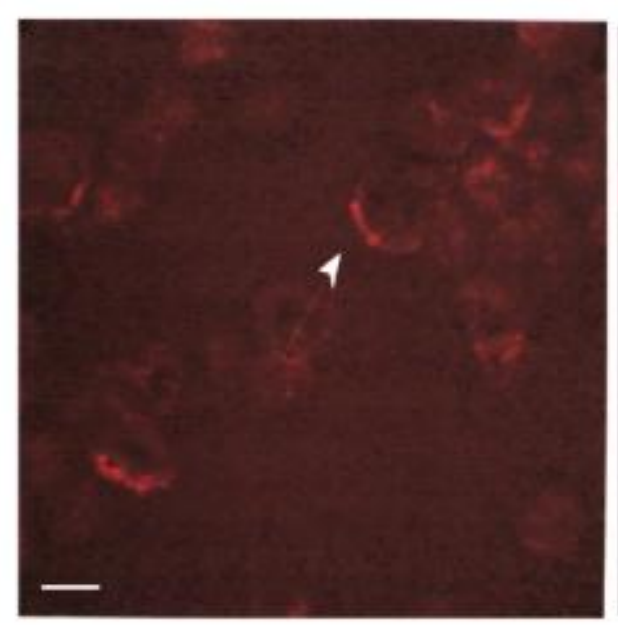

B

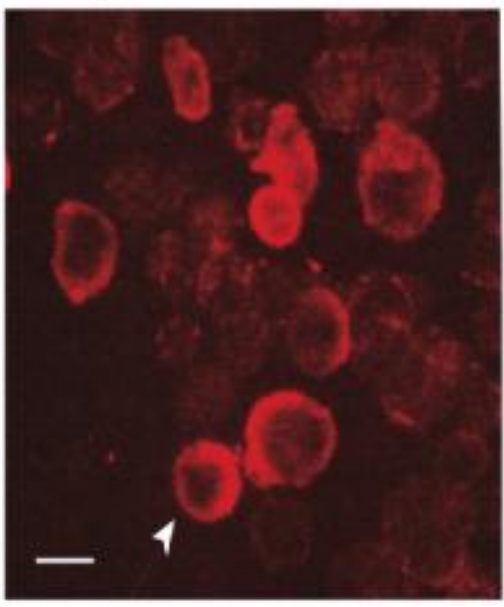

C

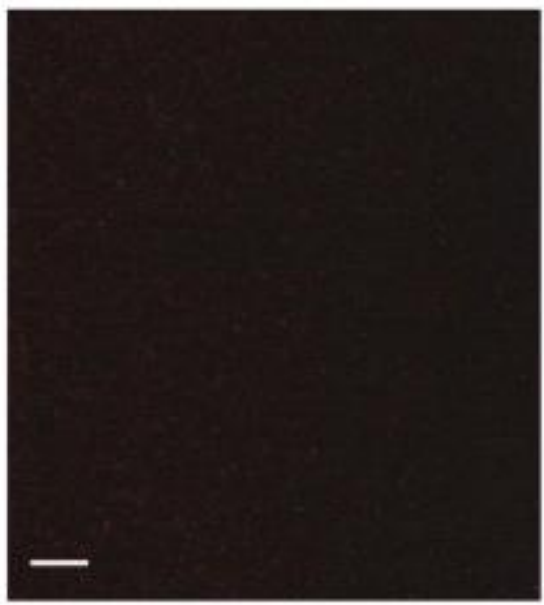

D

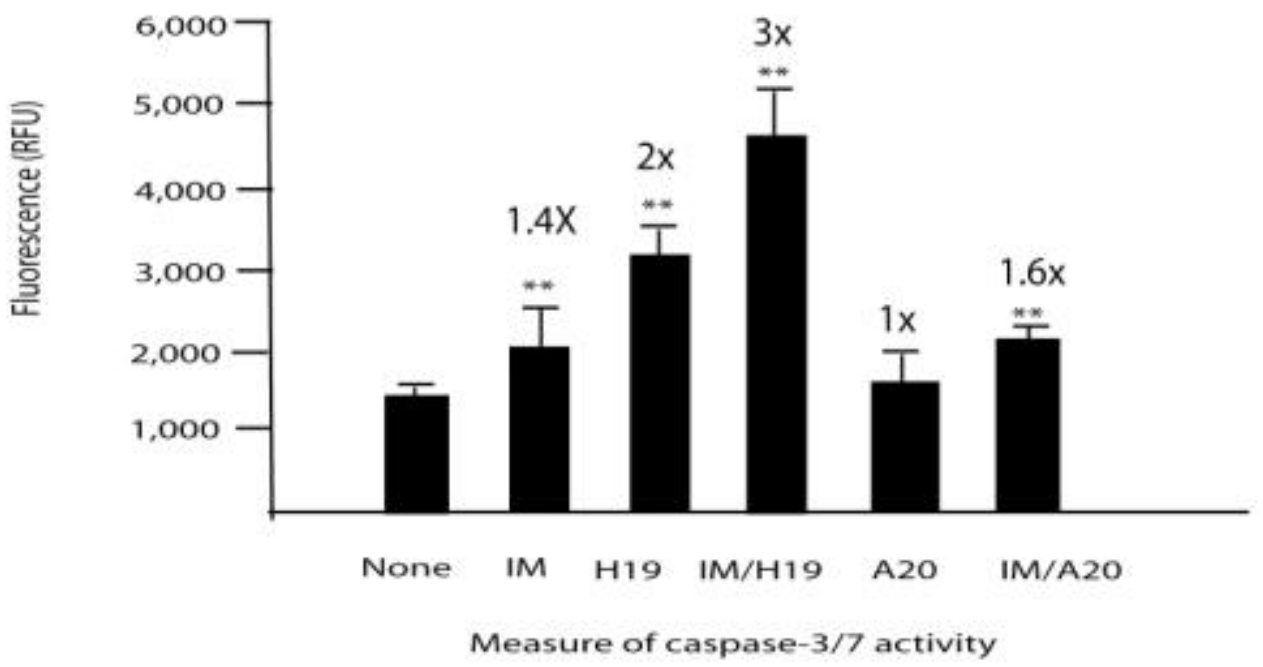

Fig. 6. Detecting surface-located GRP78 protein in PC-3 cells by immunocytohistochemistry with scFvGRP78-H19, using a second antibody against the c-myc-tag fluro555 conjugate (scale bar represents $20 \mu \mathrm{m}$ on all samples from A-C). A. PC-3 cells. B, same as A following exposure of cells to IM (100 nM) for $72 \mathrm{~h}$. C. No staining was seen with control antibody $\mathrm{scFv}-20$ under the same conditions as B. D. Caspase-3/7 activity in cell lysates was detected by activation of a fluorescent substrate. The relative florescence unit $(\mathrm{RFU})$ were presented as mean \pm standard error $(\mathrm{N}=3)$. The double asterisk indicates a significant difference $(\mathrm{P}<0.01)$ between un-treated cells and IM or antibody treated cells.

glucose-deprivation(Fig.7A). Importantly, the heat-inactive form of the antibody has no such effect (pre-treatment of the antibody at $100^{\circ} \mathrm{C}$ for 20 minutes) on the same condition. Similarly, human prostate cancer PC-3 cells were treated scFv-GRP78-H19 (83 nM) together with etoposide $(10 \mu \mathrm{g} / \mathrm{ml})$ and $2 \mathrm{DG}(20 \mathrm{mM})$, causing significant apoptotic cell death (31\%) than treatment with scFv-GRP78-H19 (15\%) or etoposide $(8 \%)$ alone after $48 \mathrm{~h}$ treatment (Fig. 7B). Again, the heat-inactive form of antibody had no effect on the same condition.

Western blotting was used to access GRP78 protein level in the above conditions in HT-29 

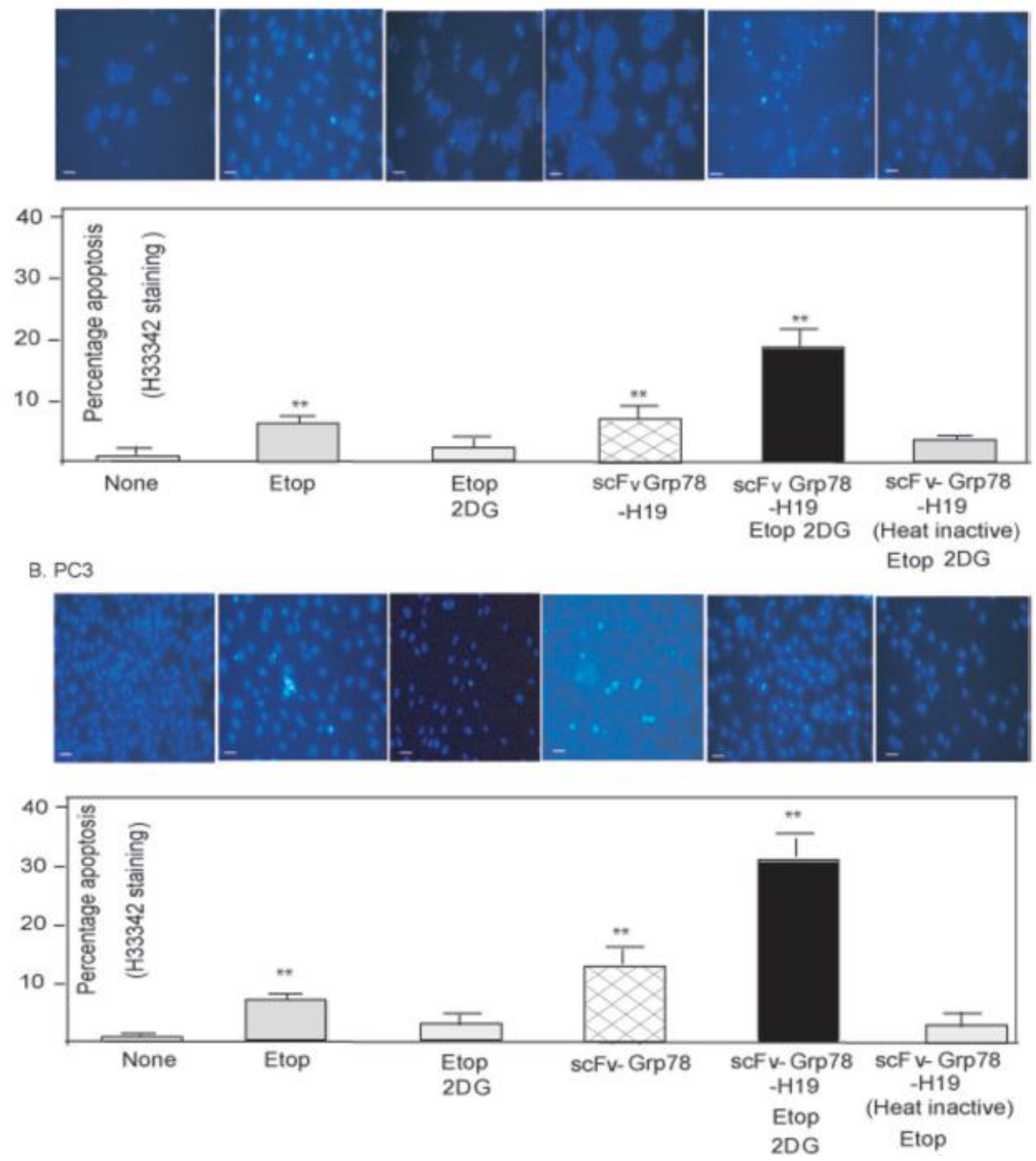

Fig. 7. A. Effects of scFv-GRP78-H19 on the apoptosis of HT-29 colon cancer cells treated with Etoposide (Etop) for $48 \mathrm{~h}$ with or without 2DG. Apoptotic nuclei or nuclear DNA strand breaks were visualized using Hoechst DNA dye H33342. Scale bar represents $20 \mu \mathrm{m}$ on all samples. Values in the graph are the mean \pm S.D. of 3 individual experiments. Double asterisk indicates a significant difference $(\mathrm{P}<0.01)$ between none treatment cells and Epotoside or antibody treatment cells. B. Effects of scFv-GRP78-H19 on the apoptosis of PC-3 prostate cancer cells treated with Etoposide (Etop) for $48 \mathrm{~h}$ with or without 2DG. Apoptotic nuclei or nuclear DNA strand breaks were visualized using Hoechst DNA dye H33342. Scale bar represents $20 \mu \mathrm{m}$ on all samples. Values in the graph are the mean \pm S.D. of 3 individual experiments. Double asterisk indicates a significant difference $(\mathrm{P}<0.01)$ between none treatment cells and Epotoside or antibody treatment cells. 
colon cancer cell line (Fig.8). We found that treating cells with 2DG alone (Fig.8, lane 3) and 2DG plus etoposide (Fig.8, lane 4) led GRP78 protein increase. However, GRP78 has significantly decreased with the ScFv-GRP78H19 antibody treatment, indicating the antibody enhanced the etoposide cytotoxicity by decreasing GRP78 protein level (Fig.8, track 5).

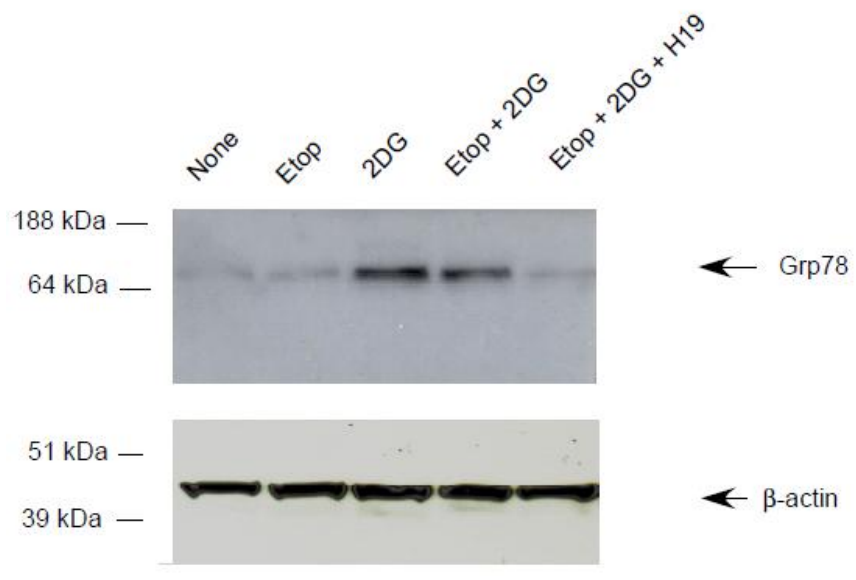

Fig. 8. Western blot analysis of GRP78 protein in Etoposide-treated HT-29 cells. Cells either untreated (none, track 1) or treated with etoposide (Etop, track 2), 2DG(track3), etoposide and 2DG(track4), etoposide, 2DG and scFv-GRP78H19 antibody (track 5). $\beta$-actin was used as a loading control and anti-GRP78 antibody was from BD. The western blot represents three independent experiments.

\section{Discussion}

Therapeutic MAbs have enormous potential for the treatment of cancer. Since 1997, the first therapeutic antibody, rituximab (Rituxan; Genetich/Biogen Idec) was approved to treat Bcell non-Hodgkin's lymphoma, there have been tremendous advance in this field and more than 17 therapeutic antibodies approved by FDA for cancer treatment[1,2]. Technical advances on the antibody based therapy have also been rapidly developed. Progress in molecular biology and immunology has allowed the derivation of specific recognition domains from MAbs. Bacteriophage encoding a single Immunoglobulin variable (V) gene -domain antibody fragment on their surface was first developed in 1990[18]. In recent years, phage display of nonimmune, human naïve single-chain $\mathrm{Fv}(\mathrm{scFv})$ or Fab antibody repertoires has proven to be an important tool for generating highly specific antibody [1.2]. In fact, most of newly developed antibodies entering clinical trails are derived from phage display technology or transgenic mice that express human immunoglobulin genes [2].

The advantage of the newly developed phage display libraries is that it contains the up to $10^{8}$ diversified and individual recombinant $\mathrm{scFv}$ fragments, which fit for high-throughput screening [15]. Identification of tumor-specific cell surface antigens is the first and critical step toward the development of targeted therapeutics for cancer, many attempts have been used in this type of phage display technology to find the tumor specific antibodies [4]. In fact, Jakobsen et al.,[19] recently described isolation of anti-GRP78 monoclonal $\mathrm{scFv}$ antibodies from a phage display library using live primary breast cancer cells. Some of them, such as Ab39 have been shown to be able to bind breast cancer surface. However, the success of direct cell selection using tumor cell has been limited. This is partly due to a high none specific binding of phage to cell surface and partly due to the high presentation of phage antibody binding to high presentation normal molecules, which present in both normal and tumor cells. The other bottle neck is subsequent proteomic work involved in identify the target antigen. In this study, we selected to use GRP78 recombinant protein as bait first for screening the phage library to eliminate the non specific binding present in the complex phage libraries, and then subjected the pooled positive phage to the cell surface GRP78 for selection. This crucial subtractive step was used to eliminate those positive GRP78 phage, which only binding to the intracellular proteins. After the cell surface selection, the positive phages were subjected to final selections using recombinant protein. These simple and alterative selections were enabling us to isolate cell surface-specific anti-GRP78 scFV antibody over 100 million different $\mathrm{scFv}$ fragments in the "naïve" or "single pot" phageantibody libraries. 
We have demonstrated that the isolated antiGRP78 cell surface targeting scFV was capable of targeting cell surface antigen and reducing endogenous GRP78 protein level. In addition, due to relative small size of the single-domain antibody and its ability to internalize, a surfacetargeting GRP78 scFv antibody could be internalize into the target cells and direct interact with cytosolic components mediating the apoptotic pathway. Our observation that antimembrane-bound GRP78 scFv dramatically increased the target cancer cell caspase-3/7 activity implicated that it could interfere with the GRP78-procaspase-7 inhibitory complex[13], a key component that provides cancer cell apoptosis-resistance against several commonly used anti-cancer drugs, such as etoposide[20].

Recently, Misra et al., [21] demonstrated that antibodies directed against the $\mathrm{COOH}$-terminal domain of GRP78 up-regulated the tumor suppressor protein $\mathrm{p} 53$, while antibody directed against the $\mathrm{NH}(2)$-terminal domain showed negligible effect on p53 expression. This study, together with our own antibody study, have highlighted the possibility that anti-GRP78 antibodies have the potential to be used therapeutically for the treatment of cancer.

The most dramatic observation of our study was that by combination the scFv-GRP78-H19 antibody and 2DG and topoisomerase inhibitors such as etoposide could dramatically increase colon and prostate tumor cell apoptosis. It is wellknown that in poorly vascularized solid tumors, where glucose deprivation and chronic anoxia are known to persist, GRP78 has been found as one of the major stress inducible proteins [12]. Indeed, GRP78 have been detected in majority of breast cancer samples [22] and in the late-stage malignant prostate cancer [23.24] and colon cancer [25]. From GRP78 transgenic experiment [26], it has been shown GRP78 protein played a major role in tumor cell proliferation and angiogenesis. More importantly, partial reduction of GRP78 in mice substantially reduced tumor cell angiogenesis without detrimental effect on normal tissue vasculature. Therefore, GRP78 acts as an ideal biomarker for tumor cell proliferation and angiogenesis. The combination treatment of GRP78 antibody and other chemotherapeutic agents such as etoposide could hold a major advance in treatment of many solid tumors, in which epotoside currently has already used as a form of chemotherapy such as Ewing's sarcoma, lung cancer, testicular cancer, prostate cancer, colon cancer lymphoma, non-lymphocytic leukemia, and glioblastoma multiform [27].

The work presented here has highlighted the possibility that anti-GRP78 antibodies have the potential to be used therapeutically for the treatment of cancer. In comparison with small interference RNA (siRNA) of GRP78 (27), scFv anti-GRP78 does not need transfection reagent. It relative small size and easily penetration of tumor tissue make it more attractive as therapeutic reagent. Finally, the subtraction antibody screening method described here has potential application for the isolation of MAbs targeting other surface-located proteins. Recently, by using biotinylation, many previously thought to be intracellular proteins such as GRP78, GRP75, HSP70, HSP60, HSP54, HSP27, and protein disulfide isomerase[10], were found to be highly abundant on the cancer cell surface, therefore warranted to be explore using alternative subtraction screening described here to identify membrane-bound antibodies for future antibodymediated cancer therapy study.

\section{Acknowledgements}

WX and LL acknowledge the support of the MRC UK. We thank Professor Ian G. Charles for his advise throughout the project. We also thank Corrado D'Arrigo and Kakinder Pal Sidhu for help screening scFv library and Alexia Pisiou for some of immunocytohistochemistry works. We are grateful to Sylvie Y. Blond for generous gift of murine GRP78 plasmid and Heather Lamb and Alastair Hawkins for providing us murine recombinant GRP78 protein. The two human scFv-phage display libraries, Tomlinson I and $\mathbf{J}$ human single fold $\mathrm{scFv}$ libraries, were kindly supplied to us by MRC Human Genome Mapping Project Resource Centre, Cambridge. 


\section{References}

1. Schrama, D.; Reisfeld, R.A. \& Becker, J.C. Antibody targeted drugs as cancer therapeutics. Nat. Rev. Drug Discov. 2006, 5, 147-159.

2. Carter, P.J. Potent antibody therapeutics by design. Nat. Rev. Immunol. 2006, 6, 343-357.

3. Ridgway, J.B.; Ng, E.; Kern, J.A.; Lee, J.; Brush, J.; Goddard, A.; Cater. P. Identification of a human anti-CD55 single-chain Fv by subtractive panning of a phage library using tumor and nontumor cell lines. Cancer Res. 1999, 59, 2718-2723.

4. Hoogenboom, H.R. Overview of antibody phage-display technology and its applications. Methods in Molecular Biology 2002,178, 137.

5. Liu, L.; Huq,S.; and Xu. W. Targeting Cyclooxygenase and Nitric Oxide Pathway Cross-Talk: A New Signal Transduction Pathway for Developing More Effective AntiInflammatory Drugs. Current Signal Transduction Therapy 2009, 4, 66-75

6. Xu, W.; Liu, L.Z.; Loizidou, M.; Ahmed, M.; Charles,I.G. The role of nitric oxide in cancer. Cell Res. 2002, 12, 311-20.

7. Xu, W.; Liu, L.; Smith, G.C.; Charles, I.G. Nitric oxide up regulates expression of DNAPKcs to protect cells from DNA-damaging anti-tumour agents. Nat Cell Biol . 2000, 2, 339- 345.

8. Xu, W.; Liu, L. and Charles, I. G. Microencapsulated iNOS-expressing cells cause tumour suppression in mice. The FASEB J. 2002, 16, 213-215.

9. Xu, W.; Liu, L.; Charles, I.G.; Moncada, S. Nitric oxide induces coupling of mitochondrial signalling with the endoplasmic reticulum stress response. Nat. Cell Biol. 2004, 6,1129-1134.

10. Shin, B.K.; Wang. H.; Yim, A.M. et al. Global profiling of the cell surface proteome of cancer cells uncovers an abundance of proteins with chaperone function. J. Biol. Chem. 2003, 278: 7607-7616.

11. Mintz, P.J.; Kim, J.; Do, K.A. et al. Fingerprinting the circulating repertoire of antibodies from cancer patients. Nat. Biotechnol 2003,21,57-63.

12. Lee, A.S. GRP78 induction in cancer: therapeutic and prognostic implications. Cancer Res. 2007, 67, 3496-3499.

13. Reddy, R.K.; Mao, C.; Baumeister, P.; Austin, R.C.; Kaufman, R.J.; Lee, A.S. Endoplasmic reticulum chaperone protein GRP78 protects cells from apoptosis induced by topoisomerase inhibitors: role of ATP binding site in suppression of caspase-7 activation. J. Biol. Chem. 2003, 278, 20915-20924.

14. Lamb, H.K.; Mee, C.; Xu, W.; Liu, L.; Blond, S.; Cooper, A.; Charles, I.G.; Hawkins, A.R. The affinity of a major $\mathrm{Ca}^{2+}$ binding site on GRP78 is differentially enhanced by ADP and ATP. J. Biol. Chem. 2006, 28, 8796-8805.

15. de Wildt, R.M.; Mundy, C.R.; Gorick, B.D.; Tomlinson, I.M. Antibody arrays for highthroughput screening of antibody-antigen interactions. Nat. Biotechnol. 2000, 18, $989-$ 994

16. Miyake, H.; Hara, I.; Arakawa, S.; Kamidono, S. Stress protein GRP78 prevents apoptosis induced by calcium ionophore, ionomycin, but not by glycosylation inhibitor, tunicamycin, in human prostate cancer cells. J. Cell Biochem. 2000, 77, 396-408.

17. Hwang, J.H.; Kim, J.Y.; Cha, M.R.; et al Etoposide-resistant HT-29 human colon carcinoma cells during glucose deprivation are sensitive to piericidin A, a GRP78 downregulator. J Cell Physiol. 2008, 21, 243-250.

18. McCafferty, J.; GriffithS A.D.; Winter, G.; Chiswell, D.J. Phage antibodies: filamentous phage displaying antibody variable domains. Nature 1990, 348,552-554.

19. Jakobsen, C.G.; Rasmussen, N.; Laenkholm, A.V.; Ditzel, H.J. Phage display derived human monoclonal antibodies isolated by binding to the surface of live primary breast cancer cells recognize GRP78. Cancer Res. 2007, 67, 9507-17.

20. Ermakova, S.P.; Kang, B.S.; Choi, B.Y, et al. (-)-Epigallocatechin gallate overcomes resistance to etoposide-induced cell death by targeting the molecular chaperone glucoseregulated protein 78. Cancer Res. 2006, 66, 9260-9269.

(C) 2009 by NWPII. All rights reserved. 
21. Misra, U.K.; Mowery. Y.; Kaczowka, S.; Pizzo, S.V. Ligation of cancer cell surface GRP78 with antibodies directed against its COOH-terminal domain up-regulates p53 activity and promotes apoptosis. Mol Cancer Ther. 2009, 8,1350-1362.

22. Fernandez, P.M.; Tabbaraso, J.; Jacobs, L.K. et al. Over expression of the glucose-regulated stress gene GRP78 in malignant but not benign human breast lesions. Breast Cancer Res. Treat. 2000, 59, 15-26

23. Arap, M.A.; Lahdenranta, J.; Mintz, P.J.; Hajitou, A.; Sarkis, A.S.; Arap, W.; Pasqualini, R. Cell surface expression of the stress response chaperone GRP78 enables tumor targeting by circulating ligands. Cancer Cell 2004, 6, 275-284.

24. Daneshmand, S.; Quek, M.L.; Lin, E.; et al. Glucose-regulated protein GRP78 is up- regulated in prostate cancer and correlates with recurrence and survival. Hum Pathol. 2007,38, 1547-52.

25. Xing, X. ; Lai ,M.; Wang, Y.; Xu, E. ;Huang, Q. Overexpression of glucose-regulated protein 78 in colon cancer. Clin Chim Acta. 2006, 364, 308-315.

26. Dong, D.; Ni, M.; Li, J. et al. Critical role of the stress chaperone GRP78/BiP in tumor proliferation, survival, and tumor angiogenesis in transgene-induced mammary tumor development. Cancer Res. 2008, 68, 498-505.

27. Pyrko, P.; Schönthal, A.H.; Hofman, F.M.; Chen, T.C.; Lee, A.S. The unfolded protein response regulator $\mathrm{GRP} 78 / \mathrm{BiP}$ as a novel target for increasing chemosensitivity in malignant gliomas. Cancer Res. 2007, 67, 9809-9816. 